\title{
To Your Good Health! Going to the Pub With Friends, Nursing Dying Patients, And 'ER' Receptionists: the Ubiquitous Rise of Risk Management and Maybe A 'Prudential' Bioethics?
}

\author{
Michael A. Ashby • Bronwen Morrell
}

Published online: 9 April 2019

(C) Journal of Bioethical Inquiry Pty Ltd. 2019

We live in an era of risk management. Whatever business you are in, your management training will now have substantial input from risk experts, who have developed a field all of their own. This is reassuring, whether we are passengers, customers, patients, or students, everybody wants to be as safe as possible. We want those working for and with us to be safety conscious, and to have safe systems in place. There are, however, significant ethical dimensions to risk management, pointing to a 'prudential' thread in bioethics, as this issue will show in several of its papers. One big question is the balance between personal liberty and harm avoidance. Another that flows from this is the question of the level of risk we accept and who has responsibility for it - there is always risk, it is never zero. Another is the danger of systems, workers, and the public becoming so risk averse that we become

\section{A. Ashby $(\bowtie)$}

Cancer, Chronic Disease and Sub-Acute Stream, Royal Hobart Hospital, Tasmanian Health Service, University of Tasmania, Repatriation Centre, 90 Davey Street, Hobart, TAS 7000, Australia e-mail: michael.ashby@ths.tas.gov.au

\section{A. Ashby}

Medical Ethics and Death Studies, School of Medicine, College of Health and Medicine, University of Tasmania, Repatriation

Centre, 90 Davey Street, Hobart, TAS 7000, Australia

B. Morrell

Sydney Health Ethics, Faculty of Medicine and Health, University of Sydney, Level 1, Medical Foundation Building, K25, Sydney, NSW 2006, Australia

e-mail: bronwenmorrell@gmail.com paralysed by our own policies, procedures and fears. Bureaucratic compliance and legal requirements end up stifling the activity they were intended to protect, and add significantly to costs of service delivery. We also see a drift away from individual clinical judgment and responsibility, to systems, policies and pathways. Such deviation can be problematical and open to censure, with compliance and documentation distracting from person-based care. The film 'Sully' ${ }^{1}$ shows this well, as a seasoned pilot saves the lives of his passengers by ditching in the Hudson River in New York, thereby deviating from the manual. He subsequently has to defend his actions in a tribunal before being exonerated. This shift from the personal to the policy manual needs examination and monitoring, lest it paralyse practitioners from the exercise of professional ethical judgement, with attendant individual responsibility.

Bioethics can help here by informing practice and jurisprudence in such a way that risk can be understood and shared by all of us, at personal, institutional and societal levels. Bioethics is deeply imbued with the investigation of individual rights and responsibilities, and hosts curated conversations about conflicting values, needs and wishes. Maybe we are seeing the emergence of a 'prudential' bioethics that focuses on the balance between personal freedom and dignity on

\footnotetext{
${ }^{1}$ The 2016 dramatic film, 'Sully', is based on the autobiography Highest Duty by Chesley Sullenberger and Jeffrey Zaslowa. Depicting the 2009 emergency landing of US Airways Flight 1549 on the Hudson River, the film was directed by Clint Eastwood, written by Todd Komarnicki, and distributed by Warner Bros (https://www. warnerbros.com/movies/sully/). Accessed 26/3/2019.
} 
the one hand, and the safety of all on the other, manifest in the modern risk management movement. There is particular need for these discussions in the care of the mentally ill and the elderly. This certainly is not new, as vulnerability and lack of mental capacity, and risk, go hand in hand.

Risk management is ultimately a proximate activity, for it is in practice and life actions that it needs to happen, not in the training or policy manual, which can too often be where effort and compliance has to be invested. This issue contains two papers that highlight risk, and both are strikingly practical in this bioethical journal setting, where complex argumentation is usually the norm. The first is a tongue in cheek exploration of the ethics that flow from a simple decision to go to the pub by our Consulting Editor and Executive Committee Member Dr David Shaw of Basel and Maastricht (Shaw 2019). The second, by a public health group in Sydney, questions the internationally accepted nursing practice of frequent regular turning of frail elderly patients to avoid pressure sores (Sharp, Shulz Moore, and McLaws 2019). Two very different papers, one a good-humoured look at some dimensions of everyday living, the other challenging the assumption about the two-hourly turning ritual in nursing care with both published empirical data and ethical reflection.

The subject of bioethics is a discourse mediated for the most part by academic papers written to explore specific complex biological issues in health and bioscience. As such, it may be seen as a remote technical exercise that is off-putting and inaccessible to those outside the tent, conducted away from the more mundane aspects of life, both personal and professional. Orientated towards the life of the mind, bioethics can tend not to look at the feet on the ground, the simple practical components of the everyday life where the plumber is often a more pressing immediate need than the philosopher. There is of course a rich matrix of realtime ethics in day-to-day life that Shaw draws attention to by opening up a rabbit hole journey of going to the pub with some friends in Scotland. There are both public health and personal health dimensions in the factors involved in different journeys undertaken to get to the pub and, as a result of different practical arrangements, the amount of alcohol that is consumed. It shows here that social conventions, in this case buying a round, can have a significant effect on health. The social, emotional, and behavioural aspects of friendship and conventions of social meeting are also rich with ethical considerations such as social exclusion and the role of personalities in determining group action. The conceptual is constantly influenced by the deeply personal and interpersonal, and, hence, the mores of a group of Scottish friends are susceptible to moral and ethical analysis, but inter-subjectivity determines what happens. Shaw's piece also shows that when seemingly simple life events are analysed more deeply, the process does indeed open up a nested set of complexities. This type of matrix thinking becomes rather like a root cause analysisthe deliberative process that has little place in everyday life. The relative roles of the logical positive in the deeper unconscious subjective personal forces that determine our everyday life decisions are rarely subjected to this type of exploration. And yet it is in the conduct of everyday life, and the practical aspects of clinical practice and scientific research, that ethics is played out, where the good and the bad actually happen. It is in individual conduct, in the personal gesture, in the individual ethical disposition, and in courtesy and consideration of the other, that bioethics is enacted in life. Ultimately these things can be logically analysed and dissected, but in life they have to be lived forwards, without much time for reflection, with the conscious and unconscious social and personal influences all wrapped up together in real-time. As Soren Kierkegaard points out, we understand life backwards but have to live it forwards. Bioethics is firmly located in the backwards understanding but aims to influence life going forwards, to create a more ethical future.

With unconscious forces, comes the inevitable shadow side of self-interest, both attraction and its shadow side of hatred, exclusion, fear, and insecurity. These are depicted in the person who Shaw's drinking group excludes without his or her knowledge, the self-interest of the chosen location, the desire for the forbidden fruit of neurochemical pleasure from alcohol, the damage that it can do in excess are all ethics-rich issues. The conflicts between the inner child that seeks gratification in dialogue with the intellect, and its more prudential and altruistic messages, are also played out in both spoken and unspoken ways between a group of friends where social exclusion and alienation nonetheless are never very far away. So too bioethics is the conscious intellectual unravelling of the threads of the rope, which to be of any use, has to be tightly entwined to be able to function. Whether it be the influence on climate change of the personal journeys we take, or the social construction of health damage, the piece reminds us that we live 
with constant contradictions, unconscious personal forces, complex social conventions, and that the toast before the first drink in many cultures is an exhortation to good health!

Care of the frail aged is the biggest challenge facing most health systems today. A prudential approach has been adopted in professional nursing practice to prevent harm. It has been woven into health service standards worldwide, with its objectives of ensuring quality improvement programs and myriad assessment forms and checklists that accompany the compliance requirements for accreditation. While having laudable aims, the result in everyday care of the elderly is a clear privileging of the physical over everything else. There is a culture of fear within nursing about pressure area and falls prevention. It is a badge of nursing honour to avoid pressure areas and if one arises under the care of a particular nurse or nursing team, the blame is certainly ascribed in the system despite all protestations to the contrary by quality improvement and safety experts. A no-blame culture maybe the aim, but it is certainly not the result of the present policy and practice environment in aged care. The imposition of a rigid systematic approach to pressure area prevention is the so-called two-hourly turn practice. It is therefore a mandated nursing practice standard in most settings. The problem is that this nursing intervention is a clinical intervention like every other medical treatment and needs to be deployed flexibly, according to the condition of the patient, proximity to death, and personal preference. In the care of the dying, in the terminal phase, quality of life and comfort should trump all other considerations, and the two-hourly turn as a nursing order should be questioned and adapted in a patient-centred care plan, where policies are indeed bent to the needs and wishes of the particular patient. Sharp and colleagues go so far as to suggest that the blind imposition of this rule, particularly when embedded as a response to accreditation standards, could even on occasions constitute elder abuse. It is this "one size fits all" rule and recipe-based care planning that they rightly question. If true holistic care is to be delivered and the system is serious about patient-centred care, then rules such as these need to be open to question and flexible individual patient-based deployment. It is all too easy for practices such as these to become unquestioned rituals, embedded in accreditation requirements, that in future times may be seen to have been unnecessary. The example of routine pubic shaves in obstetric practice springs to mind. This practice was rigidly enforced for women before giving birth before later being questioned, but eventually found to be unsupported by evidence of benefit, and stopped. Likewise Sharp et al. show that the evidence behind the practice of twohourly turning is in fact unconvincing in terms of terms of skin integrity preservation. In an era of supposedly evidence-based healthcare, it seems that dogmatic practice can still occur and deeply embedded procedures always need to be questioned.

Michaela Okninski, in the regular legal Recent Developments contribution (Okninski 2019), reports on a case that will be of interest to all who are responsible for the safe running of hospital emergency departments ('ERs'). In Darnley v Croydon Health Services NHS Trust [2018] UKSC 151, the UK's Supreme Court overturned lower court judgements, and found that a hospital trust was at fault in law for the misinformation given to a patient with a head injury. Being told of a waiting time, that turned out to be wrong, by the emergency department receptionist, a patient left the department without triage assessment. He then returned later with an extradural haematoma and required urgent brain surgery to evacuate this, with the consequence of a permanent hemiplegia, attributed to the delay in diagnosis and treatment. Safety and quality of care is an allembracing corporate responsibility of health providers, and all staff contribute to the overall safety of patients. The courts did try to understand and fairly assess the tremendous pressures on those who work in emergency medicine, and differential levels of responsibility and training depending on role, but in the final analysis the law has reinforced that it is a team effort, and 'front of house', as we all know, in any human services activity, is very important. The unfortunate reality is that good health care also works best with the participation of a reasonable patient, and on a busy night in the ER, drugs, alcohol, organic brain disturbance, and fear often make people unreasonable, and therefore vulnerable. Those who work in ER need our support and understanding, it is stressful and often makes big demands on the patience of staff. By the positing of a legal precedent, the UK courts have added another slide to the ER receptionists' training program.

In the remainder of this issue we have articles covering a range of topics from the everyday world to the conceptual world. At the applied end of this spectrum, McLennan and Moore (2019) report on an empirical examination of New Zealand District Health Board open disclosure policies for reporting of adverse events. 
They raise concerns about the significant heterogeneity between and noticeable gaps within the policies they examined. In another empirical study, Vanderschaeghe, Vandenberghe, and Dierickx (2019) analyse stakeholder perspectives on early diagnosis for Alzheimer's Disease and draw attention to the reasons patients may have for not wanting to know their results. Each of these articles has immediate practical implications of interest for both clinicians and local health policymakers.

Other articles in this issue draw our attention to the ways in which concepts impact upon the applied and how the application of these concepts can, in turn, also reshape these concepts. Davis (2019), for example, in the Australasian Association of Bioethics and Health Law John McPhee (Law) Student Essay Prize 2018, discussing the boundaries of embryo research, argues that our ideas about why we value embryos must be reconsidered before we proceed to the question of what respect should be afforded to them. Walker (2019) describes how our use of prostheses shapes our conceptualisation both of prosthetics themselves and of human bodies. Kudina (2019) discusses how the application of new technologies, in this case sex selection technologies, can shape our ideas about sex, gender, and parental responsibility. Hofman addresses how human enhancement alters constructs about health in general (Hofman 2019), Elliott proposes a feedback loop whereby changing technical capacity might drive demand (Elliott 2019). Shahvisi argues that the use of 'alternative' medicine by women is driven by the neglect of their needs by mainstream 'scientific' health services (Shahvisi 2019). Jaarsma and Gelhaus examine the policy settings in Sweden for access to ADHD treatments, and the role of narratives in this process (Jaarsma and Gelhaus 2019).

These authors all explore how various concepts impact upon each other, and, for instance, how the way we conceptualise human enhancement also shapes our understandings of health in a more general way. There is, then, a sort of circular, almost 'feedback loop' between underpinning concepts and new practices. That, as we surely already know, we are shaped by technical capacity, and the task in everyday life is to ensure mastery of our tools lest they take us to places we do not wish to be in. Herein lies a core challenge and task of bioethics: to articulate and defend values that respect the rights of human beings in the face of our own human progress, ensuring that though the genie may be out of the bottle, it does not harm us, especially the most vulnerable: bioethics as risk management and the prudential again?
Last but not least we have some changes to our associate editor (AE) line-up. We farewell and thank Zubin Master, from the Mayo Clinic, Rochester, New York, who has been AE for Science and Genetics, and wish him well. We welcome Cynthia Forlini (Neuroethics), Laura Bisaillon (Migration and crossborder justice), Neera Bhatia (Recent Developments), Lisa Anderson-Shaw (Clinical ethics ), Adrian Carter (Emerging technologies), Michael Chapman (Ethics and ageing), Danielle Couch (Public health), Alex Holden (Dentistry), Claire Horner (Reproductive ethics), and Victoria Charlton (Bioethics and policy). Our AEs are at the heart of the JBI community, and they ensure that our editorial process has the right mix of content knowledge across the broad range of bioethics. We acknowledge and thank them all, past and present, and hope they know they are all part of this international community.

\section{References}

Davis, C. 2019. The boundaries of embryo research: Extending the Fourteen-Day Rule. Australasian Association of Bioethics and Health Law John McPhee (Law) Student Essay Prize 2018. Journal of Bioethical Inquiry 16(1): https://doi. org/10.1007/s11673-018-09895-w

Elliott, C. 2019. The looping effects of enhancement technologies. Journal of Bioethical Inquiry 16(1): https://doi.org/10.1007 /s11673-018-9893-2

Hofman, B. 2019. Human enhancement: Enhancing health or harnessing happiness? Journal of Bioethical Inquiry 16(1): https://doi.org/10.1007/s11673-018-9888-z

Jaarsma, P., and P. Gelhaus. 2019. Medium-range narratives as a complementary tool to principle-based prioritization in Sweden: Test Case “ADHD”. Journal of Bioethical Inquiry 16(1): https://doi.org/10.1007/s11673-018-9884-3

Kudina, O. 2019. Accounting for the Moral Significance of Technology: Revisiting the Case of Non-Medical Sex Selection. Journal of Bioethical Inquiry 16(1): https://doi. org/10.1007/s11673-018-9891-4

McLennan S., and J. Moore. 2019. New Zealand district health boards' open disclosure policies: A qualitative review. Journal of Bioethical Inquiry 16(1): https://doi.org/10.1007 /s11673-018-9894-1

Okninski, M.E. 2019. Recent Developments: The catastrophic consequences of negligent misinformation-Darnley v Croydon Health Services NHS Trust [2018] UKSC 151. Journal of Bioethical Inquiry 16(1): https://doi.org/10.1007 /s11673-019-09909-1

Shahvisi, A. 2019. Medicine is patriarchal, but alternative medicine is not the answer. Journal of Bioethical Inquiry 16(1): https://doi.org/10.1007/s11673-018-9890-5

Sharp, C.A., J.S. Schulz Moore, and M-L. McLaws. 2019. Twohourly repositioning for prevention of pressure ulcers in the 
elderly: Patient safety or elder abuse? Journal of Bioethical Inquiry 16(1): https://doi.org/10.1007/s11673-018-9892-3

Shaw, D. 2019. Pub philosophy. Journal of Bioethical Inquiry 16(1): https://doi.org/10.1007/s11673-019-09896-3

Walker, M.J. 2019. On Replacement Body Parts. Journal of Bioethical Inquiry 16(1): https://doi.org/10.1007/s11673018-9889-y

Vanderschaeghe, G., R. Vandenberghe, and K. Dierickx. 2019.

Stakeholders' views on early diagnosis for Alzheimer's
Disease, clinical trial participation and amyloid PET disclosure: A focus group study. Journal of Bioethical Inquiry 16(1): https://doi.org/10.1007/s11673-019-09901-9

Publisher's note Springer Nature remains neutral with regard to jurisdictional claims in published maps and institutional affiliations. 